\title{
Editorial
}

\section{eCAM: A Closer Look at Clinical Analyses}

\author{
Edwin L. Cooper \\ Department of Neurobiology, David Geffen School of Medicine at UCLA, University of California, Los Angeles, \\ CA 90095-1763, USA
}

$e C A M$ continues as it began in its quest for providing linkages and revealing the interdependence of basic science and clinical analyses. If one examines the 50 most cited papers for the month of June 2009, it is difficult to determine what is purely clinical and what is basic science; there is significant overlap and blurring. To clarify, one way is to define as clinical any investigations that use exclusively humans in trials. However, using human cells in culture could be classified as an overlap between basic science and clinical investigations. One would not argue that animal models constitute basic science, or that this category would include products from animals or plants and their role in modulating disease. Taking this stance has provided me, the biologist/ immunologist, with a chance to explore those areas that (i) overlap, (ii) are biological, (iii) are immunological, and (iv) have a certain "orientation" or trajectory that renders them intimately pertinent to complementary and alternative medicine (CAM). Clinical evidence-based approaches present unique problems primarily due to human nature and are often difficult to structure, support, plan and complete. We must also examine ethical implications and what may emerge as reputable. It is not always easy to move past single-case reports, which is the base of the hierarchical evidence-based pyramid suggested by Goldrosen and Strauss (1), a paper that has been emphasized as a model as it relates to CAM and immunology and evidence-based analyses (2).

For the following analysis exemplifying the benefit of sufficiently supported CAM approaches to clinical problems, please permit me to focus on clinical analyses

For reprints and all correspondence: Edwin L. Cooper, Department of Neurobiology, David Geffen School of Medicine at UCLA, University of California, Los Angeles, CA 90095-1763, USA. published by members of the Editorial Board. These are not meant to be exclusive, nor do I intend to neglect others, but with limited space I am compelled to be brief. In fact, I hope that this discussion will inspire and stimulate us, despite our busy schedules, to take a look every month at what papers are cited. As my recollection goes, those that are mentioned in the top-50 cited papers on this current list of June 1, 2009 have already regularly appeared earlier. One can easily scroll down after accessing any one of the 50 to determine which journals have cited the paper. Even more interesting, OUP provides another service, the possibility to send e-letters that reveal all sorts of opinions from readers. As an example of what is of interest, readers are invited to view and to see what has been published and that remains on the list of 50 . Of that, a total of 16 listed papers are by members of the Editorial Board and they cover a number of subjects: those that clearly constitute basic science and those that represent clinical analyses. In the following, I have chosen to focus on the clinical area of pain and how authors have analyzed it, to illustrate such a wide array of topics of highly-cited studies, both basic and clinical, in one subject area.

According to Ma (3), it has long been accepted that acupuncture; that is, puncturing and scraping needles at certain points on the body, can have analgesic, anesthetic, as well as therapeutic use in treating various diseases. Ma underscores the international importance of this therapy that involves the nervous system, neurotransmitters, endogenous substances and Jingluo (meridians), all of which may respond to needling stimulation and electrical acupuncture. There is now an abundance of information related to the neurobiological mechanisms of acupuncture, with respect to both neural pathways and neurotransmitters/hormonal factors that mediate autonomic regulation, pain relief and other therapeutics. From early works we understand that the analgesic effects of 
electroacupuncture (EA) are mediated by opioid peptides in the periaqueductal gray, and that nitric oxide plays an important role in mediating the cardiovascular responses to EA stimuation through the gracile nucleus-thalamic pathway. In addition, we know that other substances, including serotonin, catecholamines, inorganic chemicals and amino acids such as glutamate and $\alpha$-aminobutyric acid (GABA), mediate certain cardiovascular and analgesic effects, without a clear understanding of their role. As a result, there is now increased interest in acupuncture health care, spawning further investigations in this field. Areas include processes of the sense of needling touch, transduction of needling stimulation signals, stimulation parameters and placebos. Toward higher centers, both, evidence and understanding of neurobiological processes of acupuncture research focus on recent developments of nitric oxide mediating acupuncture signals through the dorsal medulla-thalamic pathways.

Acupuncture has been analyzed from a different angle by Lim's group in a publication by Dong Lee et al. (4), where both basic and clinical approaches have been examined. Bee venom acupuncture (BVA) is often referred to as a kind of 'herbal' acupuncture. After administration, it exerts pharmacological actions from the bioactive compounds isolated from the bee venom. Moreover, there is the added benefit of a mechanical function derived from acupuncture stimulation. Dong Lee et al. declare that BVA is growing in popularity, especially in Korea, as it is used primarily for relief of pain in many diseases. To underscore its importance, this group summarized and evaluated evidence of BVA in treatment of rheumatoid arthritis and osteoarthritis. They used various computerized literature searches (e.g. PUBMED, EMBASE and the Cochrane Library), focusing on basic science and clinical trials of BVA for arthritis. Moreover, the team examined two leading Korean journals (The Journal of Korean Society for Acupuncture and Moxibustion and The Journal of Korean Oriental Medicine). They found 67 studies, 15 of which met their criteria, two of which in turn investigate the anti-inflammation and analgesic actions of BVA. Both actions were confirmed with several animal arthritic models. For clinical analyses, two randomized controlled trials and three uncontrolled clinical trials showed that BVA was effective in treating arthritis. Although the use of BVA in treating arthritis is a promising area also for future research, there is already unchallenged but limited evidence demonstrating efficacy of BVA in arthritis. Rigorous trials that include (i) large sample sizes, (ii) adequate design, (iii) optimal dosage and (iv) concentration of BVA must be employed in order to define its role, exemplifying the benefit of rigorous basic science approaches to clinical problems.
Lewith and his group (5) analyzed acupuncture in a manner that resembles that of Ma (3). Lewith et al. systematically researched the literature, examining the effect of acupuncture on brain activation as measured by functional magnetic resonance imaging and positron emission tomography. Results revealed that specific, and largely predictable, areas of brain activation and deactivation occurred when considering the traditional Chinese functions attributable to certain specific acupuncture points. As an example, points associated with hearing and vision stimulated the visual and auditory cerebral areas. Pain, however, is a complex matrix that is intimately intertwined with expectation. Thus, the authors found that acupuncture clearly affects this matrix in both specific and non-specific ways that were consistent with specific clinical effects of pain. Moreover, they observed an effect of the expectation of the relief of pain.

Using properly supported CAM to treat pain in pediatric populations has increased considerably in recent years, according to Tsao and Zeltzer (6). Usage is especially pertinent for chronic conditions such as cancer, rheumatoid arthritis and cystic fibrosis, where pain presents a significant problem. Tsao and Zeltzer assert that despite the growing popularity of CAM to treat pediatric pain, concerns about the efficacy of these interventions remain. What then are the efficacy of CAM interventions for pain symptoms in children? CAM modalities have been analyzed, as revealed in recent publications including controlled trials and/or multiple baseline studies that focused on either chronic or acute, procedural pain. Efficacy of CAM interventions was evaluated according to the framework developed by the American Psychological Association (APA) Division 12 Task Force on Promotion and Dissemination of Psychological Procedures. Results revealed that, according to these criteria, only one CAM approach (self-hypnosis/guided imagery/ relaxation for recurrent pediatric headache) qualified as an empirically supported therapy (EST). However, this did not rule out the fact that many may be considered possibly efficacious or promising treatments for pediatric pain. Once certain methodological limitations are resolved, there seems to be promising improvement for future avenues of research.

In conclusion, I would like to stress the need for more CAM approaches to clinical problems, of course recognizing the pitfalls and the rewards if sufficient support is offered. This includes adequate support and proper examination of all parameters that surround the future efficacy of a particular trial. One possible approach to study design is to consider the extensive instructions provided by OUP for designing and ultimately publishing the outcomes of successful clinical trials. For that purpose, provided herein is an excerpt from the extensively 
reworked instructions to authors on kinds of Clinical Papers that would be considered for publication: of course after appropriate peer review. eCAM will give serious consideration to peer review the following kinds of clinical papers:

1. Brief Case Reports: The brief case reports describe, in 600 words, a single interesting case. The main criterion for selection of a case is that it should address a significant question in the CAM community or enable readers to learn something. Case reports can be but do not have to be reports of rare conditions. They can report unusual presentations of more common conditions, challenging differential diagnoses, mistaken diagnoses, novel or uncommon methods of treatment or unexpected outcomes. Preferably the case should have a good illustration. Consent for publication in print and electronically must be obtained from the patient or, if this is not possible, the next of kin. (See Patients' consent and permission to publish).

2. Developed case reports with detailed, illustrative documentation: These detailed case reports are from 1500 to 2000 words. They may be longer, up to 5000 words, if a series of cases is reported or if there is a demonstrable need for a long introduction to review the literature. These reports should be accompanied by illustrative figures. $e C A M$ 's preference is for brief case reports and the longer ones will go through a strict review by the editorial office before deemed worthy to enter the review system.

3. Case reports expanded into hypotheses: These case reports are from 1500 to 2000 words or up to 5000 words if describing a series of cases. The case or cases should be strictly analyzed and a hypothesis developed.

4. Clinical studies with as strong an evidence base as possible: Clinical studies should be submitted in the form of Original Articles. Authors should note that the journal place importance on evidence. It is preferred that randomized-controled studies with double-blinded procedures are performed. If the nature of the study does not allow such study design, authors should give thorough explanation as to why this is not possible, and how they have handled the problem to minimize bias.

\section{References}

1. Goldrosen MH, Straus SE. Complementary and alternative medicine: assessing the evidence for immunological benefits. Nat Rev Immunol 2004;4:912-21.

2. Cooper EL. CAM, eCAM, bioprospecting: The 21st century pyramid. eCAM 2005;2:125-7.

3. Ma S-X. Neurobiology of acupuncture. eCAM 2004;1:41-7.

4. Lee J-D, Park H-J, Chae Y, Lim S. An overview of bee venom acupuncture in the treatment of arthritis. eCAM 2005;2:79-84.

5. Lewith GT, White PJ, Pariente J. Investigating acupuncture using brain imaging techniques: the current state of play. eCAM 2005;2:315-19.

6. Tsao JCI, Zeltzer LK. Complementary and alternative medicine approaches for pediatric pain: a review of the state-of-the-science. eCAM 2005;2:149-59. 


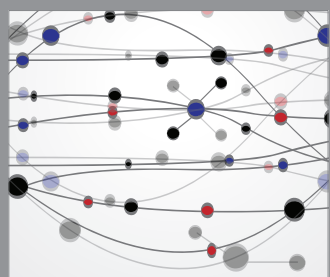

The Scientific World Journal
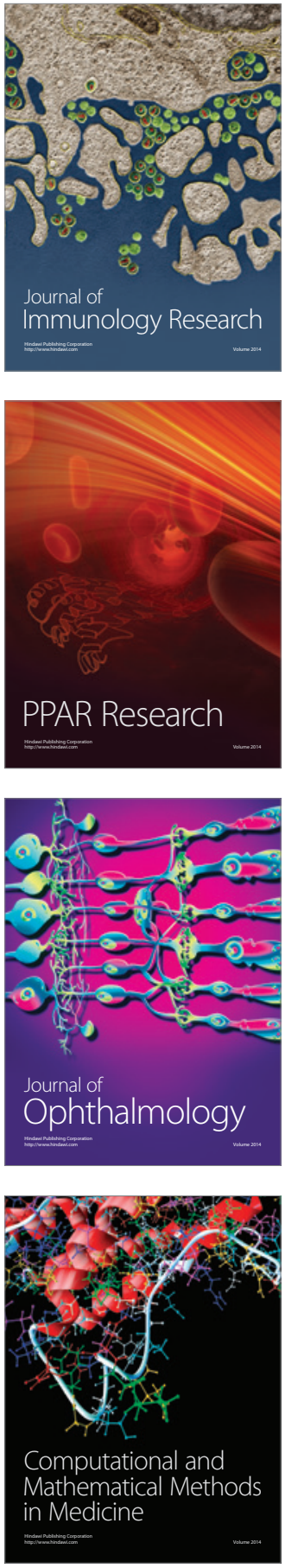

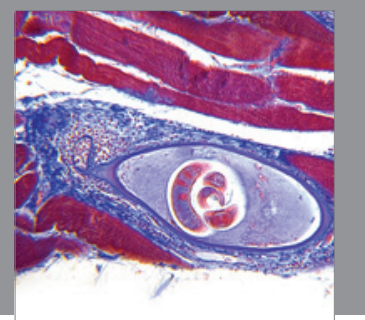

Gastroenterology

Research and Practice
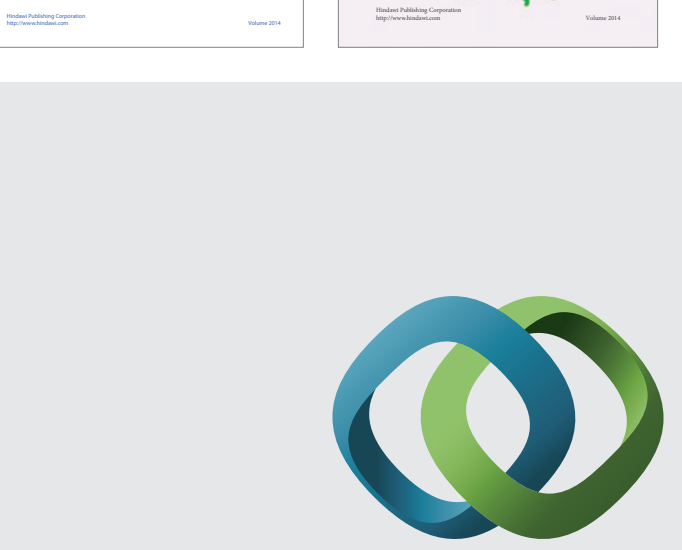

\section{Hindawi}

Submit your manuscripts at

http://www.hindawi.com
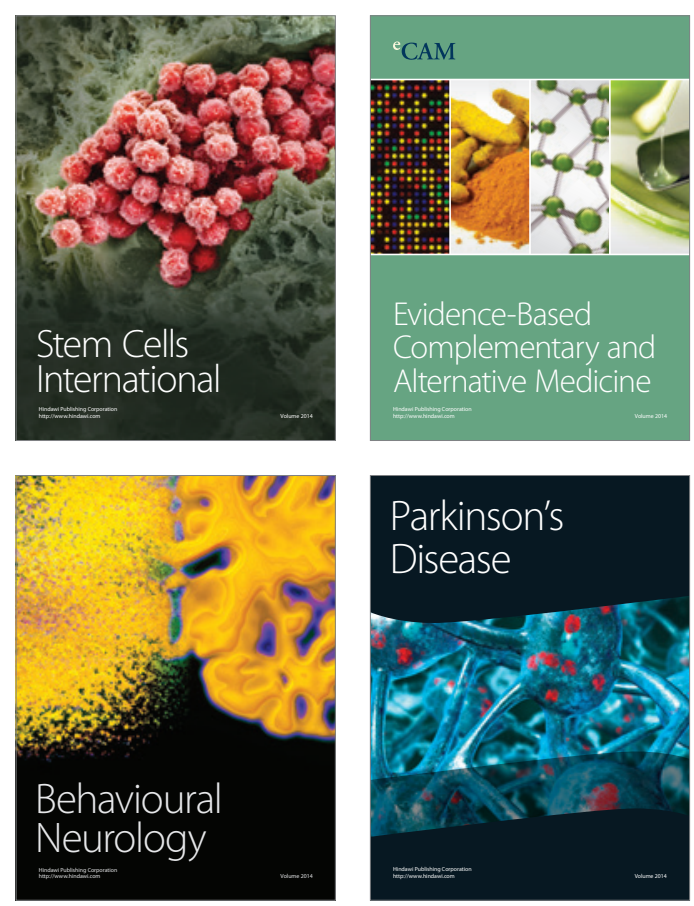

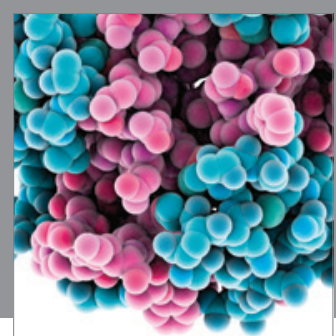

Journal of
Diabetes Research

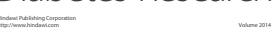

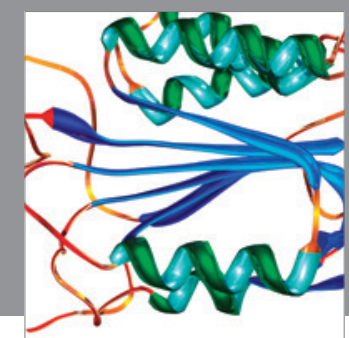

Disease Markers
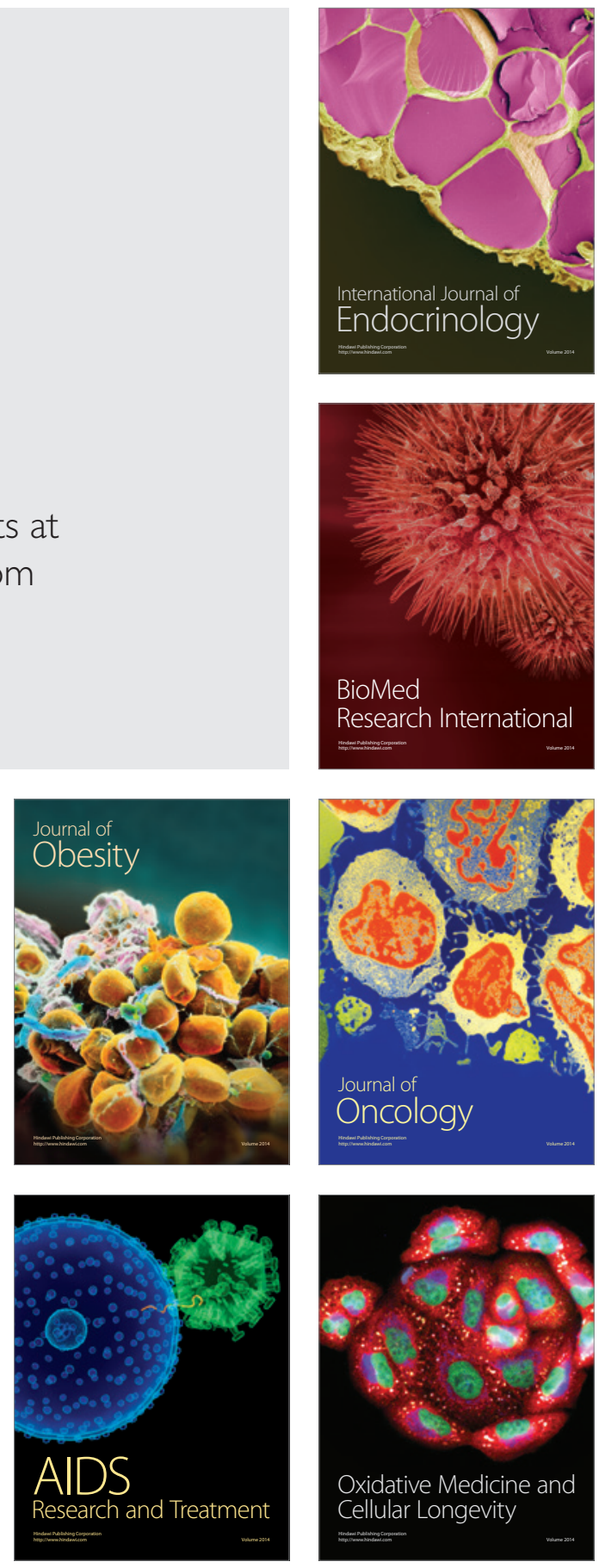